\title{
Optical and X\#Ray Properties of Cooling Neutron Stars
}

\section{Citation}

Lloyd, Don A., Lars Hernquist, and Jeremy S. Heyl. 2003. “Optical and X\#Ray Properties of Cooling Neutron Stars." The Astrophysical Journal 593 (2): 1024-31. https:// doi.org/10.1086/376589.

\section{Permanent link}

http://nrs.harvard.edu/urn-3:HUL.InstRepos:41381644

\section{Terms of Use}

This article was downloaded from Harvard University's DASH repository, and is made available under the terms and conditions applicable to Other Posted Material, as set forth at http:// nrs.harvard.edu/urn-3:HUL.InstRepos:dash.current.terms-of-use\#LAA

\section{Share Your Story}

The Harvard community has made this article openly available.

Please share how this access benefits you. Submit a story.

Accessibility 


\title{
OPTICAL AND X-RAY PROPERTIES OF COOLING NEUTRON STARS
}

\author{
Don A. Lloyd, Lars Hernquist, and Jeremy S. Heyl \\ Harvard-Smithsonian Center for Astrophysics, 60 Garden Street, Cambridge, MA 02138; dlloyd@cfa.harvard.edu, \\ 1hernquist@cfa.harvard.edu, jsheyl@cfa.harvard.edu \\ Received 2002 May 17; accepted 2003 April 28
}

\begin{abstract}
Blackbody fits to the soft X-ray spectra of cooling neutron stars will, in general, overestimate the surface temperature of the source. We examine blackbody fits to the spectra predicted by light-element neutron star model atmospheres folded with the ACIS response matrices and quantify the inferred temperature error for a range of magnetic field strengths representative of the broad populations of isolated cooling neutron stars. Results for the Position Sensitive Proportional Counter are derived for comparative purposes. In each example, the blackbody temperature systematically overestimates the underlying flux temperature of the model and is an unreliable gauge by which to estimate the surface properties of neutron stars. We demonstrate a linear relationship between flux temperature and peak energy, similar to Wien displacement, as a function of magnetic field strength for the model atmospheres. We calculate the excess optical magnitude predicted from the model atmospheres with respect to the Rayleigh-Jeans portion of the fitted blackbody. We detail the nature and magnitude of these discrepancies and identify their origin in the opacity of the atmospheric plasma, the magnitude of interstellar absorption, and the instrumental response function.
\end{abstract}

Subject headings: radiative transfer — stars: atmospheres — stars: neutron — X-rays: stars

\section{INTRODUCTION}

Models of neutron star (NS) cooling predict that they should emit thermal radiation at soft X-ray energies by an age $\tau \sim 10^{4} \mathrm{yr}$, when the core temperature has fallen to $T_{c} \sim 10^{8}-10^{9} \mathrm{~K}$ (Chiu \& Salpeter 1964; Tsuruta 1964). Thermal X-rays from NSs were first identified from ROSAT observations of nearby middle-aged radio pulsars (RPs); e.g., PSR 0656+14, PSR 0630+18 (Geminga), and PSR 1055-52 (see Ögelman 1995 for a review). Measurements of their rotation period $P$ and spin derivative $\dot{P}$ suggest these objects have characteristic ages $\tau \sim 10^{5} \mathrm{yr}$ and magnetic field strengths $B \sim 10^{12} \mathrm{G}$. Unambiguous detection of thermal radiation from most young radio pulsars is impaired by synchrotron emission originating in the pulsar magnetosphere, while many older pulsars are simply too cool to be detected as thermal sources.

The synchrotron continuum of young RPs is dominant at optical and near-UV wavelengths, and for several pulsars the continuum has been measured from radio to optical wavelengths, X-rays, and, more rarely, in $\gamma$-rays. In the middle-aged pulsars, the synchrotron amplitude is much smaller, and the transition from synchrotron to thermal emission occurs at optical or near -UV wavelengths. Several middle-aged RPs have now been detected in the optical (Mignani, Caraveo, \& Bignami 1997; Martin, Halpern, \& Schiminovich 1998; Golden \& Shearer 1999), but only Geminga has been sufficiently well studied to probe the relative contributions of the two components.

The brightest galactic X-ray sources are X-ray binaries that derive their high luminosity $\left(L_{\mathrm{X}} \sim 10^{33}-10^{38} \mathrm{ergs} \mathrm{s}^{-1}\right)$ from accretion onto a NS from its companion, typically an ordinary cool dwarf star. A small subset of these, low-mass $\mathrm{X}$-ray binaries (LMXBs), exhibit long periods of quiescence during which $L_{\mathrm{X}}$ is reduced by a factor of 3-5 from that of its outburst phase, and thermal emission from the NS surface can be detected. Thermal X-rays from the (quiescent) LMXBs are probably produced either from a very low rate of accretion onto the NS surface during quiescence or from reheating of the stellar crust during massive accretion events (Brown, Bildsten, \& Rutledge 1998). In quiescence, optical emission from the binary system is primarily that of the companion star, making its spectroscopic classification possible but obscuring optical emission from the NS surface.

By the mid-1990s, a new class of X-ray pulsars emerged from ROSAT and EXOSAT observations (Mereghetti \& Stella 1995), which, unlike the X-ray binaries, does not appear to be accretion-powered. These "anomalous" X-ray pulsars (AXPs) are characterized by an X-ray luminosity well in excess of their spin-down luminosity ( $L_{\mathrm{X}} \sim 10^{35}-10^{36} \mathrm{ergs} \mathrm{s}^{-1}$ ), the absence of radio emission, long spin periods (6-12 s), and stable long-term spin down. Models for these sources have invoked accretion from a low-mass, low-luminosity secondary (Mereghetti \& Stella 1995; Stella, Israel, \& Mereghetti 1998) or from a circumstellar disk (van Paradijs, Taam, \& van den Heuvel 1995; Chatterjee, Hernquist, \& Narayan 2000) as the X-ray production mechanism. The former model is increasingly untenable given the strong constraints placed on the secondary by IR, optical, and UV observations (Hulleman, van Kerkwijk, \& Kulkarni 2000), while the disk model requires a fairly contrived set of physical parameters to reproduce the observed X-ray spectra but is not otherwise ruled out by observation (see, e.g., Menou, Perna, \& Hernquist 2001). Spectral fits to the X-ray data for these stars generally require both a nonthermal (power-law) component and a thermal one.

An alternative explanation may be found in the "magnetar" model (Duncan \& Thompson 1992; Thompson \& Duncan 1996; Heyl \& Kulkarni 1998) in which the X-ray luminosity is powered by magnetic field decay in the crust of ultramagnetized NSs $\left(B \sim 10^{14}-10^{15} \mathrm{G}\right)$, which heats the stellar core. In the magnetar picture AXPs, like the middleaged RPs, emit cooling radiation from the stellar surface at $\mathrm{X}$-ray energies. The high luminosity of the sources suggests that the photosphere should consist of light elements 
$(\mathrm{H} / \mathrm{He})$ rather than a more opaque iron plasma. The search for optical counterparts (either from a secondary circumstellar disk or from the NS surface) is made difficult by the large typically absorbing columns through which these sources are observed $\left(N_{\mathrm{H}} \sim 10^{22} \mathrm{~cm}^{-2}\right)$, and it is for this reason that the presence of a main-sequence companion has not be unequivocally ruled out for all AXPs (Mereghetti et al. 2001). The absence of radio emission from the AXPs may suggest that if the magnetar picture is correct, these NSs can be expected to emit thermal radiation at optical wavelengths. However, the only optically identified AXP counterpart, $4 \mathrm{U} 0142+61$, is inconsistent with each of the current models for the X-ray emission.

The AXPs are a subset of the larger category of radioquiet neutron stars (RQNSs) or "inactive" radio pulsars, which includes isolated NSs (e.g., RX J185635-3754) and a few examples found in supernova remnants (Cas A, Pup A, and RCW 103/1E 161348-5055). Many of these sources are poorly understood in terms of their spin properties and magnetic field strength because of the difficulty of measuring either radio emission, modulation of the thermal X-rays, or, in the case of RCW 103, the origin of long-term brightness fluctuations (Gotthelf, Petre, \& Vasisht 1999).

Interpretation of the thermal emission from isolated NS can provide insight to basic properties of the stellar surface: temperature, composition, and effective emitting area, which are otherwise inaccessible from the synchrotron continuum alone. The spectrum of a hot, light-element NS atmosphere differs substantially from a blackbody because of the energy dependence of the free-free absorption coefficient; the X-ray spectrum of such an atmosphere is the sum of photons emitted not from an isothermal source but from a plasma atmosphere possessing a temperature gradient. In particular, the peak emission of the Planck spectrum occurs at a lower energy than that of a light-element atmosphere having the same total flux. Furthermore, the shape of the blackbody peak differs from that of a NS model spectrum, the latter being somewhat broader because of emergent radiation originating from a continuous range of temperatures in the atmosphere while retaining the characteristic Rayleigh-Jeans (RJ) behavior of the blackbody in the opti$\mathrm{cal}$ and near-UV. This is not strictly true of the magnetar models, in which the plasma opacity to optical and UV wavelengths is sensitive to collective effects at high densities; this issue must be addressed by future efforts. Consequently, the predicted flux ratio $f_{\mathrm{X}} / f_{\mathrm{opt}}$ and optical magnitude deduced from blackbody fits to thermal NS spectra will exceed observed limits while, for a given source distance, the stellar radius is underestimated.

The Planck function, parameterized only by the flux temperature, has the advantage of simplicity for spectral fitting. More realistic spectra predicted from model atmosphere calculations are further parameterized by the magnetic field strength and geometry of the star, the composition of the atmospheric plasma, and the equation of state for the plasma constituents. The calculation of thermal spectra from isolated NSs requires greater effort in both computational expense and analysis of degeneracies in the model parameters, but these efforts can be rewarded with a more robust description of the nature of the stellar surface; the Planck function alone is an unreliable measure of these properties.

To study the nature and magnitude of these discrepancies, we have computed mock X-ray spectra from light-element model atmospheres for a range of tempera- tures and magnetic fields representative of the NS populations described previously. We derive a correlation between the peak energy and flux temperature from the models. Blackbody fits to the mock spectra were made to evaluate the magnitude of the temperature error $T_{\mathrm{bb}} / T_{\text {eff }}$. We have also computed the optical magnitude excess of the model spectra, measured with respect to the magnitude derived from the blackbody fit. Some qualitative remarks on the consequences for the optical measurements of thermally emitting RPs and the AXPs will be made in $\S 4$.

\section{THE MODELS}

We have calculated model atmospheres in radiative equilibrium for effective temperatures $\log T_{\text {eff }}=5.5-6.5$ and magnetic field strengths $\log B=8.0-14.0$ for pure hydrogen in the limit of complete ionization. Unmagnetized model atmospheres have also been computed for each value of $T_{\text {eff }}$. All models assume a neutron star of mass $1.4 M_{\odot}$ having a $10 \mathrm{~km}$ radius. The emergent spectrum is calculated for a range of photon energies from the optical to soft X-rays. For this preliminary analysis, we take the emergent intensity pattern of a single element of the stellar surface, with the magnetic field uniform and vertical, to be representative of the integrated surface emission of the NS. We will defer a more complete and detailed description of the global emission properties of NSs until a future paper.

Each model calculation proceeds from a trial solution for the temperature profile of the atmosphere, derived from an approximate power-law model for the conductivity of the plasma (Heyl \& Hernquist 1998b). This solution has the correct behavior at large depth, where the diffusion limit provides a good description of the radiation field. The trial solution is completed with a formal calculation of the radiative transfer equations for the input thermal structure. This solution is self-consistent but will not, in general, satisfy the constraints of the model and is iteratively refined using the method of complete linearization (Mihalas 1978) until the largest temperature correction $\Delta T / T \leq 0.1 \%$ and the departure from the conserved total flux is $\Delta F / F \leq 0.1 \%$ at all depths. A detailed discussion of the computational methods used to calculate the model atmospheres is in preparation.

The spectrum from each model is written in the XSPEC table model format using standard routines in the HEASARC and FITSIO software packages (Arnaud 1996). This integrated spectrum can be arbitrarily normalized for the following analysis, although the precise normalization is required to compute the optical magnitude difference as described in $\S 3.1$.

We generate mock spectra from each table model in XSPEC using the atable and fakeit commands. To quantify the influence of interstellar absorption, we consider the unabsorbed NS spectrum and spectra that include a fixed absorbing column $($ phabs $), N_{\mathrm{H}}=(0.01-1.0) \times 10^{22} \mathrm{~cm}^{-2}$, with the default interstellar absorption model. All spectra are subject to a gravitational redshift correction $Z=0.306$ to compute the flux as seen by a distant observer. We do not include additional fitted components, such as a power law. The resulting spectral model is folded with the response matrices of the ROSAT Position Sensitive Proportional Counter (PSPC) instrument and the Chandra ACIS CCD in both front-illuminated (ACIS-I) and back-illuminated (ACIS-S3) configurations to produce a mock spectrum of 
approximately 3000 counts. We then fitted an absorbed blackbody (bbodyrad), with the same $N_{\mathrm{H}}$ as the underlying model, to the mock spectrum and compute the temperature discrepancy $T_{\mathrm{bb}} / T_{\text {eff }}$. Note that this ratio is evaluated in the observer's frame, where $T_{\text {eff }}^{\infty}=(1+Z) T_{\text {eff }}$. Finally, a calculation of the magnitude difference between the spectral model and blackbody fit in the $U, V, B$, and $K$ bands is made.

Because of the relative breadth of the NS thermal peak as compared with the blackbody, for even the modest count statistics of our mock spectra, the XSPEC fits cannot simultaneously reconcile both the peak energy of the NS model and be made commensurate with the flux amplitude away from the peak. In the soft X-rays, substantial residuals persist for the best-fit blackbody, with unacceptable fits $\left(\chi^{2}>1.0\right)$ for many pairs of NS spectra and response functions. Fit quality worsens for heavily absorbed model spectra. Broadly speaking, the $\chi^{2}$ statistic grows to exceed unity linearly with $T_{\text {eff }}$ for unabsorbed model spectra; however, $\chi^{2}$ grows faster with $N_{\mathrm{H}}$ for low $T_{\text {eff }}$ models.

\section{RESULTS}

The plasma opacity includes free-free absorption and coherent scattering from electrons and protons. Magnetic corrections to these opacity sources are evaluated in the cold plasma limit (Ventura 1979; Mészáros 1992) but include the protons in the plasma dielectric tensor. Resonant scattering in electron and proton cyclotron lines is also included.

In a magnetized plasma, radiation propagates in two "normal" modes of polarization, which can have very different refractive indices. Outside of the cyclotron resonance, the angle-averaged plasma opacity to radiation in the ordinary mode is approximately that of an unmagnetized plasma, while the opacity of the extraordinary mode is smaller by a factor $\simeq\left(\omega_{c, e} / \omega\right)^{2}$, where $\omega_{c, e}$ is the electron cyclotron frequency. Radiation in the two modes will decouple from the atmosphere at different strata, with the photosphere of the extraordinary mode at a temperature greater than that of the ordinary mode. Consequently, (1) the total emergent radiation of strongly magnetized NS atmospheres can acquire a large net polarization, and (2) the shape of the emergent spectrum is principally determined by the extraordinary mode.

Figure 1 illustrates the temperature discrepancy $T_{\mathrm{bb}} / T_{\text {eff }}$ for a grid of model NS spectra of temperature $\log T_{\text {eff }}=5.5-6.5$ in steps of 0.1 and magnetic field strength $\log B=8.0-14.0$ in steps of 1.0. Unmagnetized model results are also shown for comparison with weakly magnetized models. Models with $\log B=14$ and $\log T_{\text {eff }}<6.2$ are poorly converged and have been excluded from this diagram. The $T_{\mathrm{bb}} / T_{\text {eff }}$ derived for unabsorbed spectra do not differ significantly from the $N_{\mathrm{H}}=10^{20} \mathrm{~cm}^{-2}$ results and are not shown. Because of the coarse spacing in $\log B$, resonant absorption lines appear at soft X-ray energies only for models with $\log B=11$ and 14 . The electron cyclotron feature at $1.2 \mathrm{keV}$ in the $\log B=11$ models delineates the onset of magnetic field corrections to the plasma opacity. Spectra for models in which $\log B=14$ possess a strong proton cyclotron feature at $0.6 \mathrm{keV}$ that nontrivially affects the blackbody fit parameters and statistics; the implications of this feature for the AXP spectra will be discussed below.

We expect that the $T_{\mathrm{bb}}$ derived from a genuine spectrum can be approximately related to the $T_{\text {eff }}$ of the model atmospheres, particularly if the magnetic field of the stellar surface is deduced from measurement of $P$ and $\dot{P}$ and
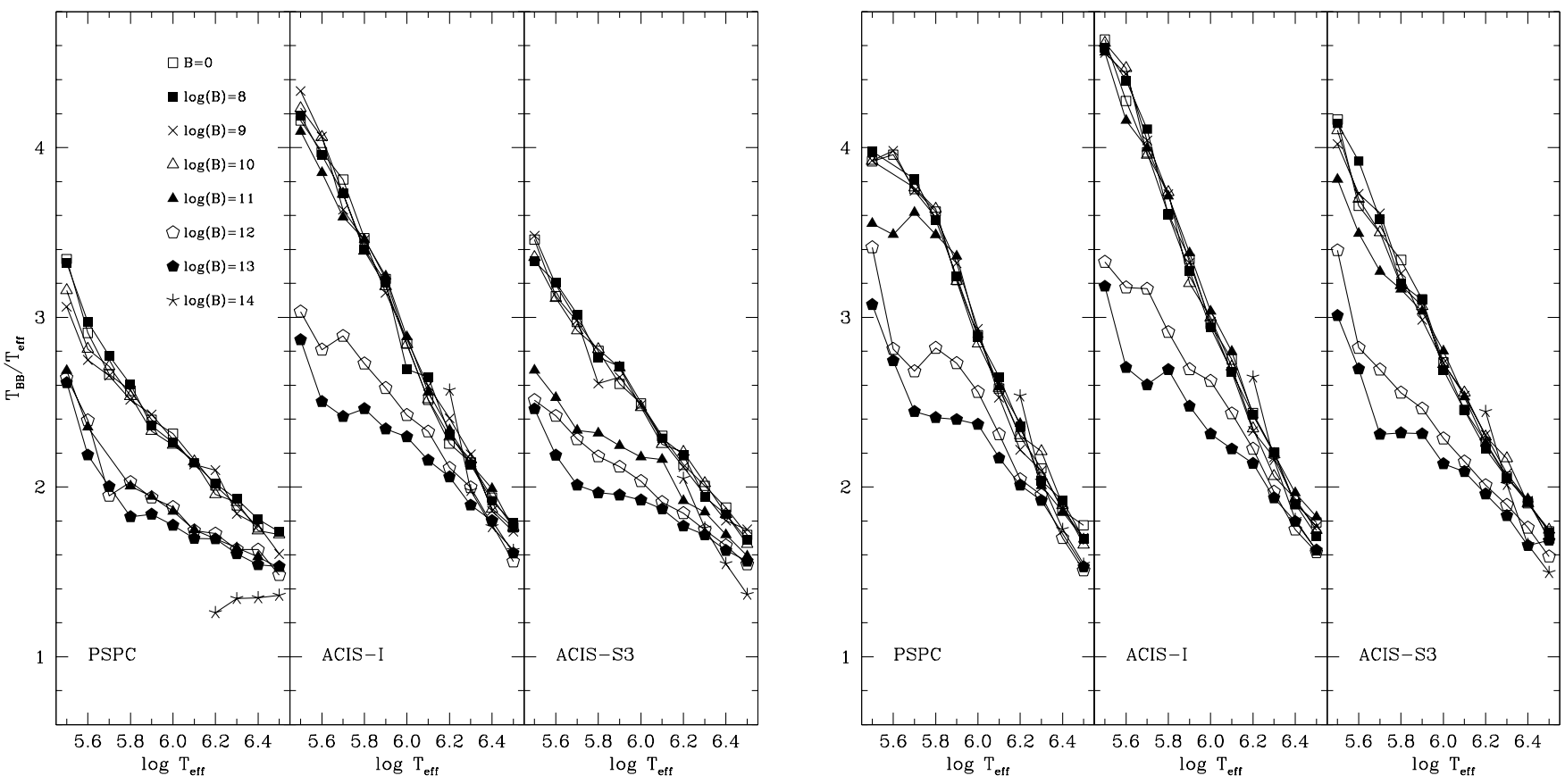

FIG. 1.-Temperature discrepancy $T_{\mathrm{bb}} / T_{\text {eff }}$ vs. $\log T_{\text {eff }}$ derived from PSPC and ACIS response matrices for column densities $N_{\mathrm{H}}=10^{20} \mathrm{~cm}^{-2}(l e f t)$ and $N_{\mathrm{H}}=10^{21} \mathrm{~cm}^{-2}$ (right). The ratio $T_{\mathrm{bb}} / T_{\text {eff }}$ is always evaluated in a common frame (e.g., for a distant observer). The X-ray spectra of model atmospheres with $B=10^{14} \mathrm{G}$ differ substantially from a blackbody because of a strong proton cyclotron resonance at $0.6 \mathrm{keV}$. Unabsorbed model results do not differ significantly from those of $N_{\mathrm{H}}=10^{20} \mathrm{~cm}^{-2}$. Heavily absorbed models, $N_{\mathrm{H}}=10^{22} \mathrm{~cm}^{-2}$, cannot be distinguished by magnetic field strength (see Fig. 4). The locus of fixed blackbody temperatures are hyperbolae in the $T_{\mathrm{bb}} / T_{\text {eff }}$ vs. $T_{\text {eff }}$ plane. See text for discussion. 

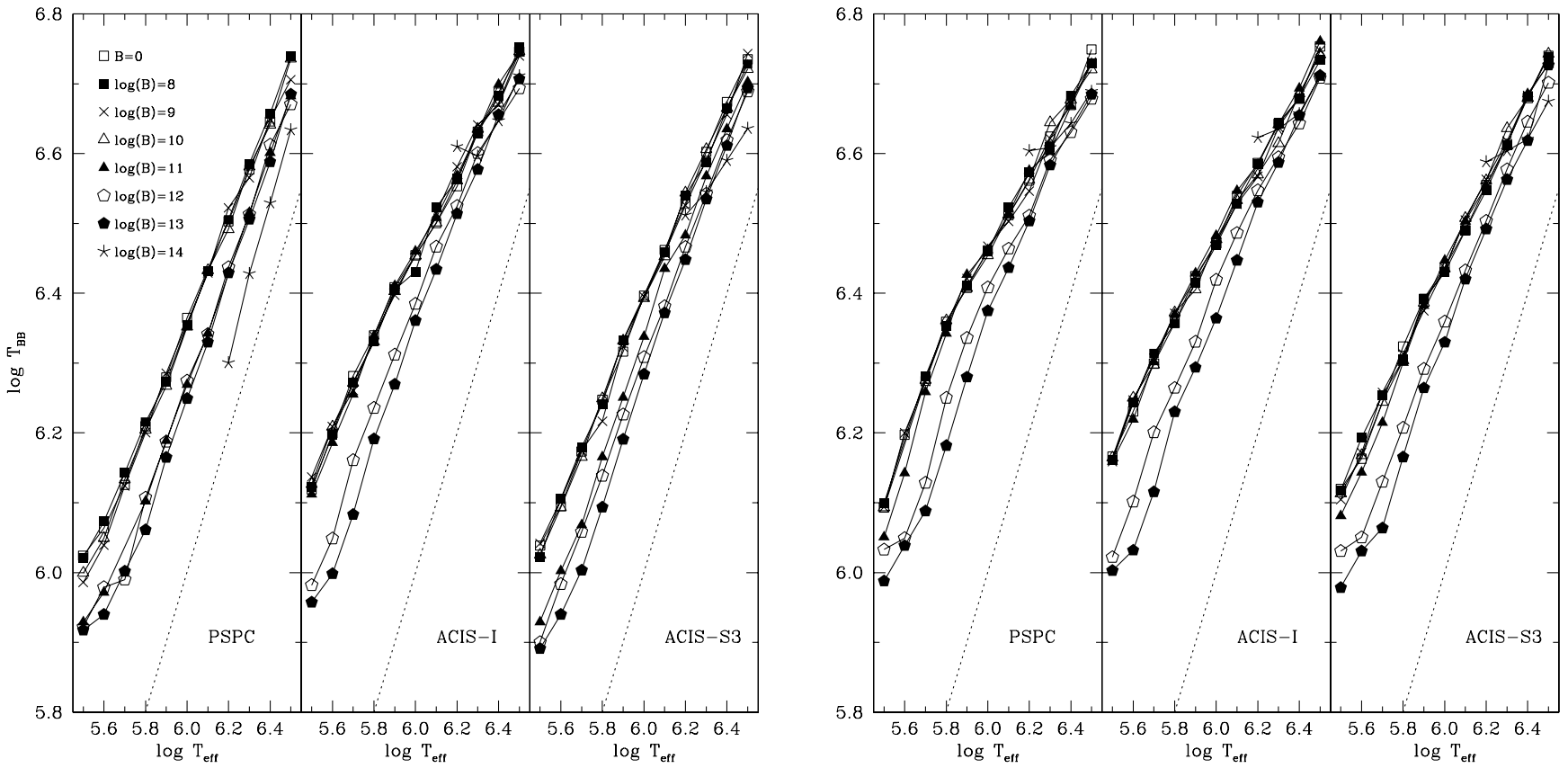

FIG. 2.- Model and blackbody pairs of Fig. 1, shown here for $T_{\mathrm{bb}}$ and $T_{\mathrm{eff}}$ in their stellar frame values for $N_{\mathrm{H}}=10^{20} \mathrm{~cm}^{-2}(l e f t)$ and $10^{21} \mathrm{~cm}^{-2}($ right $)$. The displacement of $\log T_{\mathrm{bb}}$ from the dashed $\operatorname{line}$ is $\log \left(T_{\mathrm{bb}} / T_{\text {eff }}\right)$ for both the stellar and observer frames.

assuming magnetic dipole braking. Curves of constant $T_{\mathrm{bb}}$ are hyperbolae in the coordinates of Figure 1. To facilitate estimation of $T_{\text {eff }}$ from $T_{\mathrm{bb}}$, the results of Figure 1 are shown in coordinates more useful for this purpose in Figure 2.

Unmagnetized and magnetized tracks.-Magnetic corrections to the plasma opacity become significant when the electron cyclotron frequency $\omega_{c, e}$ exceeds the characteristic thermal photon frequency. Consequently, weakly magnetized atmospheres are indistinguishable from the field-free case in both spectral profile and net polarization; in the following discussion we will refer to these as unmagnetized models. Highly magnetized NSs have somewhat redder spectra than their unmagnetized counterparts (at the same $\left.T_{\text {eff }}\right)$ because of the hard-energy dependence of the magnetized free-free absorption coefficient. We expect that blackbody fits to magnetized models will agree slightly better (i.e., result in lower $T_{\mathrm{bb}} / T_{\text {eff }}$ ) than for fits to unmagnetized models.

The onset of magnetic opacity corrections can clearly be seen in Figure 1. The models are roughly clustered into two groups, the unmagnetized track $(\log B \lesssim 10)$ and the magnetized track $(\log B>10)$, which is distinguished from the former by systematically smaller values for $T_{\mathrm{bb}} / T_{\text {eff }}$ for all $T_{\text {eff }}$. Apart from the obvious separation of weakly and strongly magnetized models, the high-field results are further delineated by magnetic field strength because of the factor $\left(\omega_{c, e} / \omega\right)^{2}$ in the magnetic absorption coefficient. For either instrument, the tracks tend to converge at the highest $T_{\text {eff }}$ of our grid.

Wien displacement.-The peak energies $E_{\gamma, \max }^{\mathrm{atm}}$ of our model spectra are roughly proportional to $T_{\text {eff }}$ for each value of $\log B$ over the order of magnitude in effective temperature we have considered (Fig. 3). This relation is similar to the Wien displacement of the blackbody function; however, for each magnetic field strength, the proportionality coefficient derived from the model atmosphere exceeds that of the Wien relation: $E_{\gamma, \text { max }}^{\text {atm }} \simeq(3.0-4.7) k T_{\text {eff }}$. Models for which $\log B=11$ possess a broad electron cyclotron feature that adversely affects the determination of $E_{\gamma, \max }^{\mathrm{atm}}$, and the correlation here is less robust. We may reasonably expect

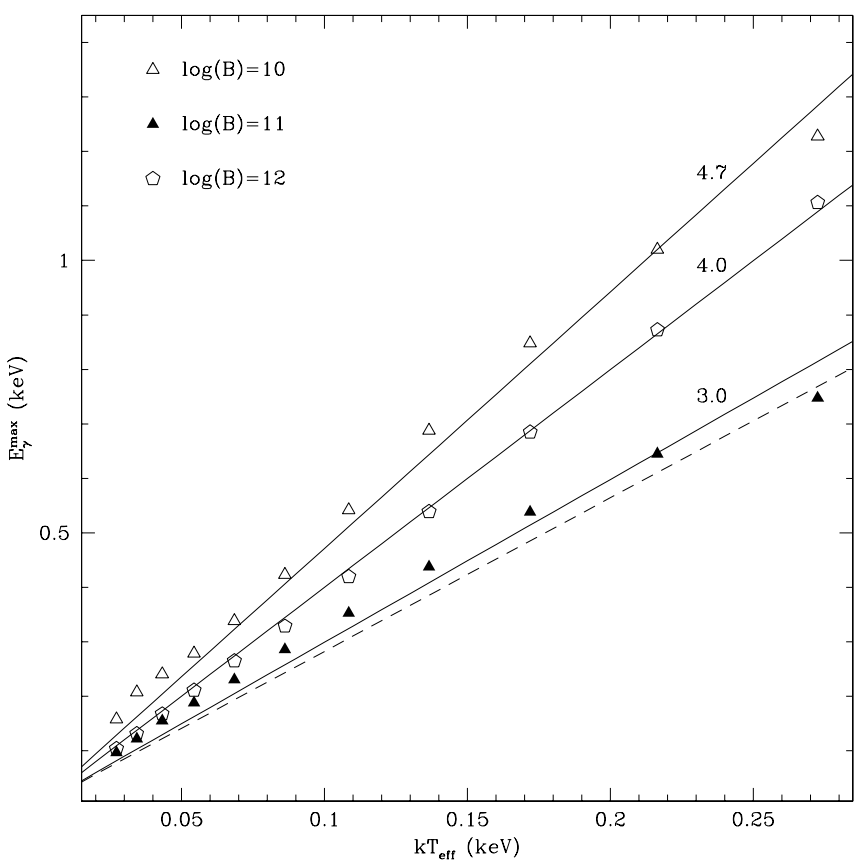

FIG. 3.-Peak energy as a function of flux temperature $T_{\text {eff }}$ for magnetic field strengths covering the transition from unmagnetized model spectra $(\log B \lesssim 10)$ to completely magnetized regime $(\log B \gtrsim 12)$. Models of $\log B=11$ have a strong electron resonance at $\sim 1.2 \mathrm{keV}$ that affects the shape of the spectral peak. Models for $\log B=14$ are similarly affected by the proton resonance $(0.6 \mathrm{keV})$, spoiling a simple linear relationship. Bestfit lines of the form $E_{\gamma}^{\max } \propto k T_{\text {eff }}$ are labeled by slope. The Wien displacement for blackbody spectra has a slope of 2.8 (dashed line). 
that while the fitted $T_{\mathrm{bb}}$ will overestimate the true flux temperature $T_{\text {eff }}$, the magnitude of the error will decrease as $T_{\text {eff }}$ increases because of the faster growth of $E_{\gamma, \max }^{\mathrm{atm}}$ with $T_{\mathrm{eff}}$. This behavior is confirmed in Figures 1 and 2 .

Departures from monotonic descent of $T_{\mathrm{bb}} / T_{\text {eff }}$ with $T_{\text {eff }}$ are not generally significant and only reflect the uncertainty in the ratio $T_{\mathrm{bb}} / T_{\mathrm{eff}}$ for the random counts distribution derived from the NS model atmospheres in XSPEC. We have not made a full study of the range of $T_{\mathrm{bb}} / T_{\text {eff }}$ that can be obtained from repeated sampling of the mock spectra.

Interstellar absorption.-Depletion of low-energy photons by absorption in the ISM reshapes the spectral envelope, shifting the peak emission to energies higher than for unabsorbed spectra. The effect of interstellar absorption is most serious on very cool models, for which the thermal peak may be significantly eroded in a strongly absorbed line of sight. Spectral fits to such cool spectra should be considered with particular skepticism because of the combined effects of absorption and instrumental response. The trend of $T_{\mathrm{bb}} / T_{\text {eff }}$ with $T_{\text {eff }}$ steepens with increasing density in the absorbing column for $N_{\mathrm{H}} \gtrsim 10^{20} \mathrm{~cm}^{-2}$, and for $N_{\mathrm{H}}=10^{22} \mathrm{~cm}^{-2}$ the distinction between unmagnetized and magnetized models is lost (Fig. 4).

Cyclotron lines.-NS models for $\log B=11$ have a "dual" character, clustering with the magnetized models when folded with PSPC and with the unmagnetized models when folded with ACIS-I; for ACIS-S3, the $T_{\mathrm{bb}} / T_{\mathrm{eff}}$ drift from the magnetized track (zero absorption) to the unmagnetized track folded with increasing absorption. This results from the combined effects of the electron cyclotron line at $\sim 1.2 \mathrm{keV}$ and the efficiency of the various response matrices. Comparison of the unabsorbed flux for models of $\log B=10-12$ at several temperatures suggests that while the peak energy of a $\log B=11$ atmosphere is more characteristic of the magnetized models, the amplitude of the high-energy tail corresponds to that of the unmagnetized
NS model at the same $T_{\text {eff }}$. The mock spectrum folded with each detector reveals that the PSPC will minimize residuals with greater emphasis on the shape of the peak and yield $T_{\mathrm{bb}} / T_{\text {eff }}$, which are consistent with their more strongly magnetized counterparts. ACIS-I favors smaller residuals in the high-energy tail and will yield temperature discrepancies similar to those for the unmagnetized models.

The results for $\log B=14$ depart from the magnetic field classification identified from the other models and are significantly affected by the strong proton cyclotron line at 0.6 $\mathrm{keV}$. At this field strength, vacuum corrections to the plasma opacity are significant and produce very soft emission at high energies, compared with the less strongly magnetized examples. The principal effect of the vacuumcorrected opacity is the formation of a heating layer in the atmosphere due to enhanced absorption of the primary flux component ( $X$-mode) (Lai \& Ho 2002; Lloyd 2003). This layer of elevated temperature is itself a resonant phenomenon, and the plasma density at which it forms is approximately quadratic in photon energy. An important auxiliary consequence of reemission by the heating layer is that the photospheric temperature of the proton line is dependent on the $T_{\text {eff }}$. For $\log T_{\text {eff }} \lesssim 6.5$ the proton absorption line acquires cusplike wings because of the enhanced emission of the heating layer, whereas the cyclotron core itself forms in cooler, less dense strata; by $\log T_{\text {eff }} \sim 6.2$ the formation depth of the core coincides with the heating layer, producing enhanced emission in the cyclotron line. Therefore, above a factor of 2.5 in $T_{\text {eff }}$, the proton cyclotron feature is inverted from the usual resonant absorption signature to an effective emission line (Lloyd 2003). This process is also responsible for the narrowing of the proton line feature. In these examples, definition of the spectral peak is ambiguous because of its proximity to the proton resonance and the $T_{\text {eff }}$ dependence on the form taken by the cyclotron feature.
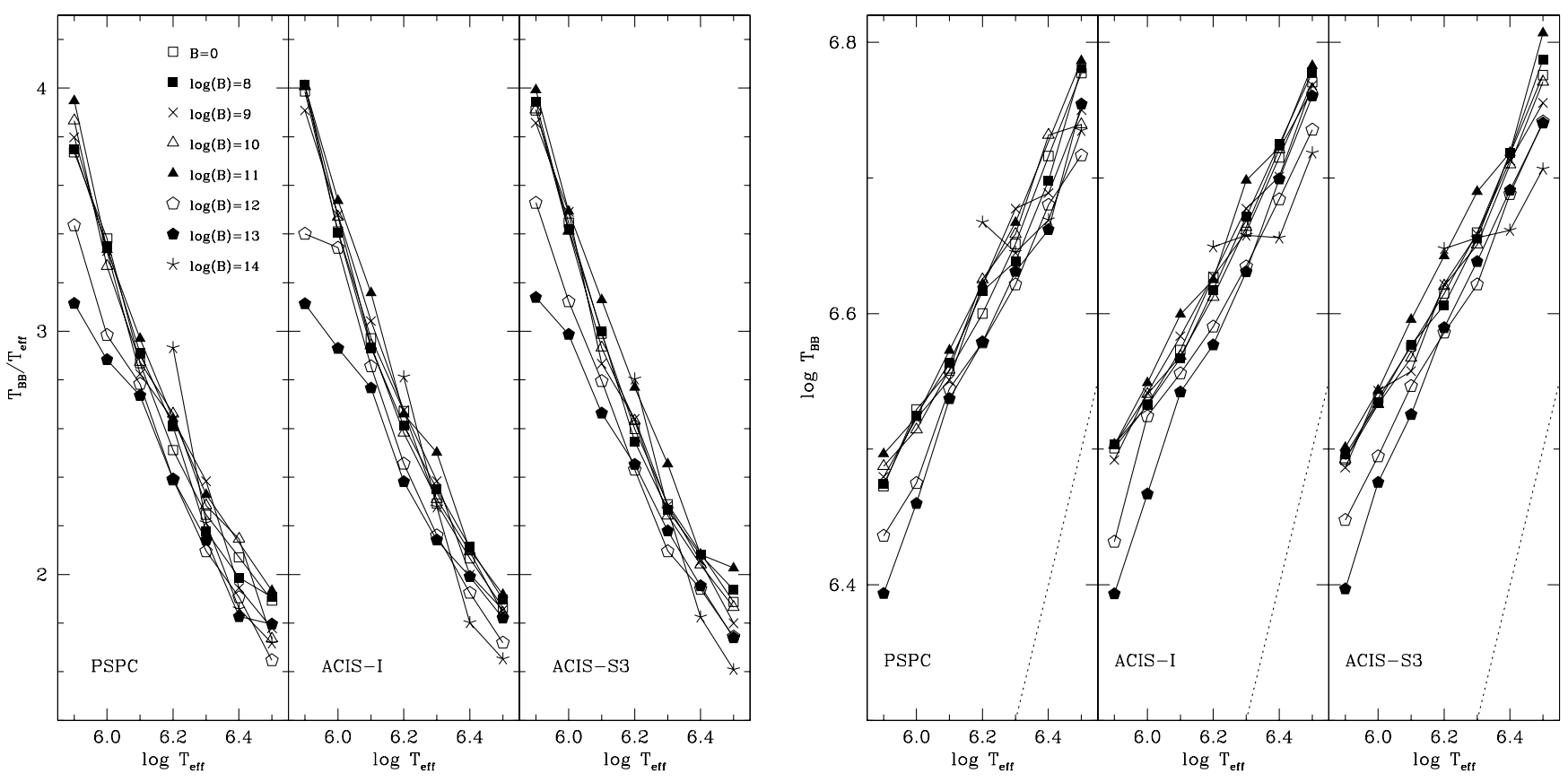

FIG. 4.- Model and blackbody pairs for heavily absorbed models $\left(\log N_{\mathrm{H}}=22\right)$ for $\log T_{\text {eff }} \geq 5.9$. The trends previously discussed for lower absorbing column densities are present, but the variation with magnetic field is nearly indistinguishable for hot NSs that could be observed with large $N_{\mathrm{H}}$. 
In general, large interstellar absorption diminishes the importance of the cyclotron feature when fitting the mock spectra, and the resultant fits tend to favor alignment of the blackbody peak to the blue "knee" of the heavily absorbed model spectra. For $N_{\mathrm{H}} \gtrsim 10^{21} \mathrm{~cm}^{-2}$, the relation between $T_{\mathrm{bb}} / T_{\text {eff }}$ and $T_{\text {eff }}$ is steeper for $\log B=14$ than for lower magnetic field strengths, regardless of instrumental response. The mock spectra produced with the PSPC for $N_{\mathrm{H}} \lesssim 10^{21} \mathrm{~cm}^{-2}$ fare poorly in this regard since its response function favors the neighborhood of the cyclotron feature; consequently, the fitted results do not immediately suggest a reliable correlation between $T_{\mathrm{bb}} / T_{\text {eff }}$ and $T_{\text {eff }}$.

\subsection{Optical Magnitude Displacement}

The $V$-band magnitude difference, $\Delta m_{V} \equiv m_{V}^{\text {atm }}-m_{V}^{\text {bb }}$, for each pair of NS mock spectrum and blackbody fit is presented in Figure 5. The magnitude of reddening $A_{V}$ for each value of $N_{\mathrm{H}}$ will have equal effects on both the spectrum and blackbody and is neglected in this analysis. Note that the underlying model is systematically brighter than the fitted blackbody for all but the PSPC result at $\log T_{\text {eff }}=6.5$ and $\log B=13$. The $\Delta m_{V}$ decline with increasing $T_{\text {eff }}$; better agreement between $T_{\mathrm{bb}}$ and $T_{\text {eff }}$ corresponds to a smaller departure of the blackbody spectrum from the NS model in the optical, although this is not intuitive. Models with similar X-ray properties can evidently have very different magnitude displacements at optical wavelengths, and simple classification of magnetized and unmagnetized models is no longer adequate.

From our discussion of $T_{\mathrm{bb}} / T_{\text {eff }}$ we found that the demarcation of magnetized models occurs at field strengths $10^{10}-10^{11} \mathrm{G}$ and is dependent on instrumental effects. The $\Delta m_{V}$ computed for these field strengths are displaced to brighter magnitudes than models with $B<10^{10} \mathrm{G}$, which are otherwise indistinguishable. At $10^{10} \mathrm{G}$, the electron cyclotron line centered at $E_{\gamma} \sim 0.12 \mathrm{eV}$ induces enhanced emission at longer wavelengths to maintain the constant radiative flux - this effect is smaller at high $T_{\text {eff }}$, where the line feature has a higher formation depth than that of a typical thermal photon, and there is consequently less radiation subject to resonant absorption. The models for $10^{11} \mathrm{G}$ are displaced to brighter magnitudes than their clustering tendencies derived from the soft X-ray spectra would suggest. The explanation for this is that the broad electron absorption feature strongly affects both the blackbody temperature and amplitude. The energy redistribution from the resonance to lower energies also contributes to the enhanced optical flux.

We previously noted that, as the magnetic field strength increases from $10^{10}$ to $10^{12} \mathrm{G}$, the plasma opacity to soft $\mathrm{X}$-rays changes from its unmagnetized value to the harder energy dependence induced by the magnetic field. The spectral envelope is particularly broad for strongly magnetized models $\left(10^{12}-10^{13} \mathrm{G}\right)$, and in general, blackbody fits to these mock spectra require larger amplitudes to minimize residuals over the soft X-ray band. From these considerations we expect that models for $\log B \geq 12$ will have small $\Delta m_{V}$ compared with those with weaker fields, particularly at high $T_{\text {eff }}$. Two additional effects can be considered. First, at $10^{12}$ $\mathrm{G}$, radiation absorbed in the broad proton resonance at $\lambda_{\infty} \sim 2570 \AA$ is redistributed to longer wavelengths, enhancing the optical flux. This is identical to the reasoning applied to the $10^{10} \mathrm{G}$ models, and similarly, the $V$-band magnitude displacement is small for high $T_{\text {eff }}$. The second effect is the growth of the plasma opacity at optical wavelengths previously described. At $10^{13} \mathrm{G}$, this flux is steeper than the Rayleigh-Jeans form common to both the blackbody and the hydrogen atmospheres for $B \lesssim 10^{13} \mathrm{G}$.
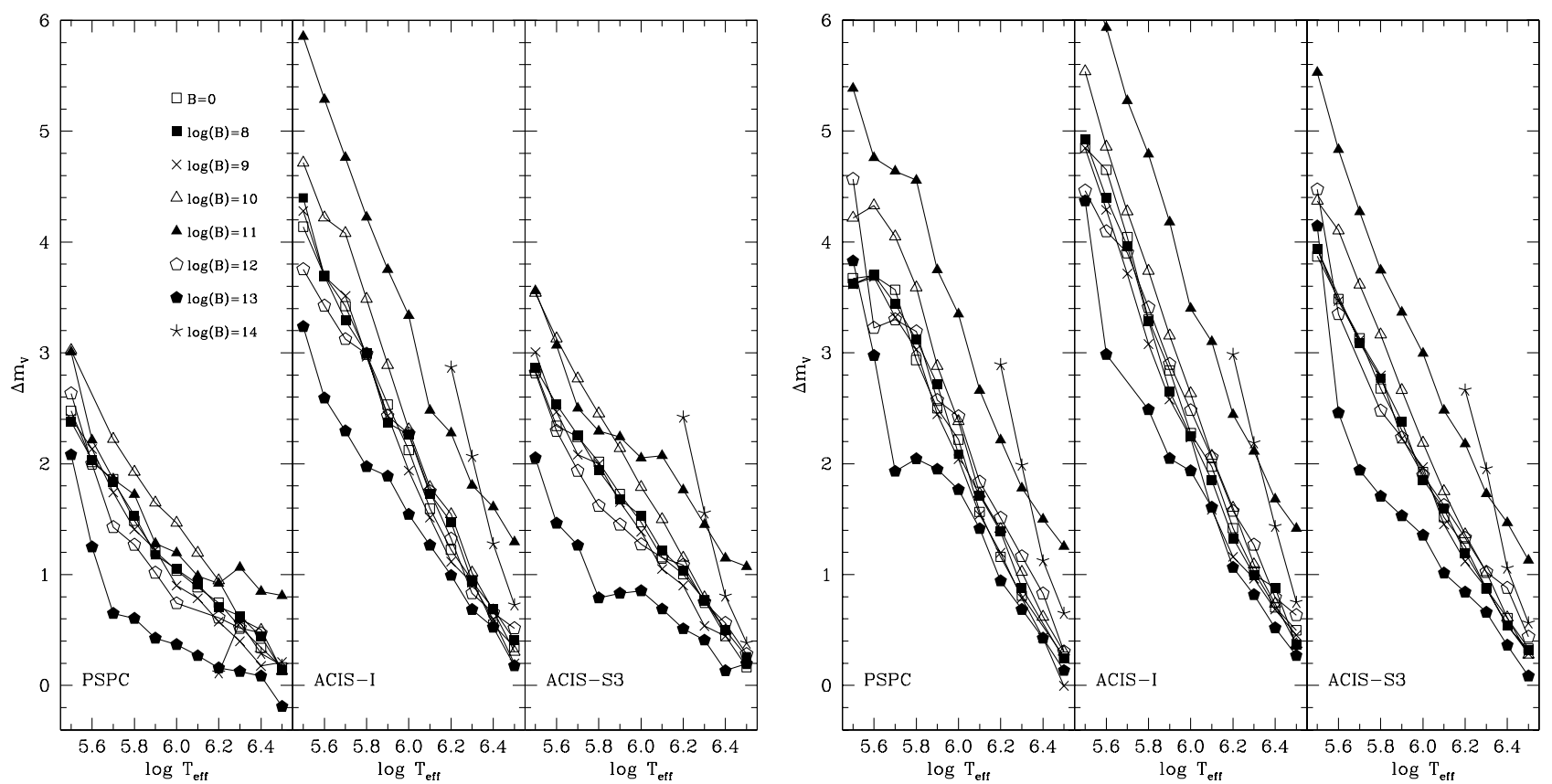

Fig. 5.-Shows $V$ magnitude displacement computed from the model blackbody pairs of Fig. 1; $B=10^{14} \mathrm{G}$ models have been excluded. The clustering of models with magnetic field regime seen in Fig. 1 persists at optical frequencies, with exceptions generally resulting from flux redistribution at energies below the cyclotron resonance. Specific cases are discussed in $\S 3.1$. 


\section{DISCUSSION}

We have quantified the discrepancy between fits to model NS X-ray spectra with the Planck function, and the underlying flux temperature, by the ratio $T_{\mathrm{bb}} / T_{\text {eff }}$ for a grid of model hydrogen atmospheres characteristic of several manifestations of cooling NSs. For an ionized light-element plasma atmosphere, the temperature $T_{\mathrm{bb}}$ obtained from a blackbody fit to the cooling NS X-ray spectra is not representative of the flux temperature. The temperature $T_{\text {eff }}$ may be estimated from tabulations of the ratio $T_{\mathrm{bb}} / T_{\text {eff }}$ by instrument, the effective Wien relation as derived from model atmosphere calculations, or obtained directly from a fit to the NS atmosphere model. The light-element NS models predict smaller surface temperatures and larger radii than the blackbody (for a fixed distance to a given object) and finite corrections to the thermal contribution at optical wavelengths from that of the RJ spectrum. Furthermore, the temperature discrepancy is substantial enough to necessitate the reinterpretation of the cooling history of a given source, either for the age of the source, or the processes by which the stellar core can cool.

Our discussion is restricted to the special case of fully ionized hydrogen plasma. In strong magnetic fields, the hydrogen ground-state binding energy grows asymptotically with $\sim \ln ^{2} B_{c}$ ryd, where $B_{c}=B / 2.35 \times 10^{9} \mathrm{G}$ (Louden 1959); Pavlov et al. (1995) have shown that even a small neutral fraction in the atmosphere can significantly alter the spectrum because of bound-free transitions from these tightly bound states.

Rajagopal \& Romani (1996) and Rajagopal, Romani, \& Miller (1997) evaluated the ratio $T_{\mathrm{bb}} / T_{\mathrm{eff}}$ for iron atmospheres and for the hydrogen atmosphere models of Pavlov et al. (1995) using 3000-count mock PSPC spectra and an absorbing column of $N_{\mathrm{H}}=10^{20} \mathrm{~cm}^{-2}$. Our results for $10^{12} \mathrm{G}$ (Fig. 1, left panel) are in good agreement with their result over the range of $T_{\text {eff }}$ given (cf. $B_{12} \equiv B / 10^{12} \mathrm{G}=1.2$ of their Fig. 5). We also find reasonably good agreement in $T_{\mathrm{bb}} / T_{\text {eff }}$ of our unmagnetized hydrogen atmosphere calculations for $\log T_{\text {eff }}<6.2$, while for higher $T_{\text {eff }}$ the mild slope of our unmagnetized track introduces departures of about $10 \%$ from their values. Our results are likewise generally consistent with their findings for $B_{12}=4.7$, where $\log T_{\text {eff }} \geq 5.7$. The bound-free transitions from neutral hydrogen become a significant opacity source below this temperature in the models of Pavlov et al. (1995), and our results for $T_{\mathrm{bb}} / T_{\text {eff }}$ are consequently discrepant with those of Rajagopal et al. (1997) for these examples.

More sophisticated models of the NS surface emission will consider the nonuniform temperature distribution that results from the anisotropic conductivity of the stellar crust (Heyl \& Hernquist 1998a). For a simple dipole field geometry, the magnetic field varies in both strength and orientation across the NS surface, inducing a smooth variation in local effective temperature. Gravitational defocusing, which enlarges the total surface area exposed to detection, must also be included (Page 1995). Detailed calculations including these effects are in progress (Lloyd 2003).

Of particular interest is measurement of the $M / R$ for neutron stars, which provides constraints on the equation of state for the supernuclear material of the NS core. The mass and radius enter our calculations through the gravitational redshift of the spectrum and through the surface gravity $g_{s}$ assumed for calculation of the model atmospheres. The model spectra have only a weak dependence on $g_{s}$ for reasonable values of $M$ and $R$, and the effect is limited to radiation emitted in the Wien tail. Emission in the Wien tail grows slightly with increased $g_{s}$ because of larger electron densities at the photospheric layers for these high-energy photons. Under realistic circumstances, the modest variation in the amplitude of the Wien emission is likely to be degenerate with the power-law component typically required for acceptable spectral fit quality.

The evolution of net polarization across the electron cyclotron feature $(\S 3)$ results from the transition from the unmagnetized at higher energies to the magnetized opacity at lower energies. The polarization of the magnetized NS $\mathrm{X}$-ray emission is manifest in the shape of the spectral envelope, as evidenced by the $\log B=11.0$ models. At this time, the polarization content of the X-ray spectrum has no particular diagnostic value. For $B \gtrsim 10^{9} \mathrm{G}$, optical emission from the NS surface is highly polarized and almost completely so at $B \simeq 10^{12} \mathrm{G}$. Optical polarimetry, therefore, may one day provide a useful probe of the properties of the NS magnetic field while confirming the mutual origin of both optical and thermal X-ray emission.

Detection of a cyclotron feature in thermal NS spectra constitutes a direct measurement of the magnetic field strength at the stellar surface. This possibility is of particular interest for the magnetars, where resonant absorption by protons should be observable at $\sim 0.5-5.0 \mathrm{keV}$ for magnetic fields on the interval $B=10^{14}-10^{15} \mathrm{G}$, assuming the canonical NS mass and radius. The nondetection of such lines in the spectra of the AXPs suggests that either the narrow-line features are at the ACIS resolution limit (Lai \& Ho 2002), that the magnetar picture does not fully describe the thermal emission from these sources, or that the magnetic field strength of these objects is overestimated in the conventional oblique rotator picture.

\subsection{Middle-aged Radio Pulsars}

A careful analysis of the combined X-ray and optical for any particular source is beyond the intended scope of this paper and may be readdressed in a future study. We limit our discussion here to qualitative remarks on the status of these observations and their interpretation with regard to the NS atmosphere model results we have described.

Of the very few radio pulsars seen as soft X-ray sources, several are optical pulsators for which at least some fraction of their optical flux is nonthermal and presumably originates in the pulsar magnetosphere: Vela, PSR 0540-69, PSR 0656+14, and Geminga. Of these, Vela and PSR 0540-69 have a negligible thermal contribution to their respective optical flux, being too bright at these wavelengths for their distance.

There is not yet a clear consensus on the relative contribution of thermal emission to Geminga's optical flux, despite its being the best studied of these sources. Many phenomenological models based on early optical studies have been presented in the literature and have invoked proton cyclotron emission (Bignami, Caraveo, \& Paul 1988; Jacchia et al. 1999) superposed on a Rayleigh-Jeans spectrum. The simplest model that satisfies most presently available data is a synchrotron continuum plus the RJ spectrum (Martin et al. 1998), which is comparable to other optically bright RPs. The optical spectrum PSR $0656+14$ is similar to that 
of Geminga and has a slightly lower $\nu_{\mathrm{eq}}$ (Pavlov et al. 1996). Our results for the optical magnitude excess described in $\S 3.1$ indicate that the synchrotron and thermal fluxes of these stars will contribute equally to the total at a lower frequency $\nu_{\mathrm{eq}}$, when the X-ray flux is modeled with a realistic atmosphere, than for a blackbody.

Three other RPs have been detected at optical frequencies for the first time with the Hubble Space Telescope, although in a limited number of filters: PSR 1055-52 (Mignani et al. 1997), PSR 0950+08, and PSR 1929+10 (Pavlov et al. 1996). Of these sources, the counterpart to PSR 1055-52 appears most compatible with thermal emission from the stellar surface. Mignani et al. (1997) found the F342 band flux for PSR 1055-52 to be about $0.5 \mathrm{mag}$ fainter the extrapolated blackbody fit to the ROSAT data (Ögelman \& Finley 1993), having assumed a source distance $d=500 \mathrm{pc}$, smaller than that suggested by previous radio data to constrain the size of the NS surface. Given that our models predict an optical excess to the blackbody spectrum for the observable radio pulsars, the F342 flux can be made consistent with a model atmosphere spectrum only if the source distance is smaller than $500 \mathrm{pc}$.

\section{2. $A X P 4 U 0142+61$}

Recent Chandra X-ray observations of the AXP $4 \mathrm{U}$ $0142+61(d \geq 2.7 \mathrm{kpc})$ are best fitted by a two-component model including a power-law contribution $(\Gamma=3.3)$ in addition to a soft thermal source $\left.T_{\mathrm{bb}}=4.8 \times 10^{6} \mathrm{~K}\right)(\mathrm{Juett}$ et al. 2002), properties typical of the other AXPs. In the magnetar scenario, the soft X-ray component is emitted from the surface of the NS while the nonthermal flux presumably originates in the stellar magnetosphere. Preliminary calculations of pulse properties for a dipole magnetic field configuration with a nonuniform temperature distribution for such a NS (Lloyd 2003) suggest that the thermal $\mathrm{X}$-rays alone can account for the $7.5 \%$ pulse fraction observed at $1 \mathrm{keV}$ (Israel et al. 1999).

Unlike the other members of its class, $4 \mathrm{U} 0142+61$ has a well-defined optical counterpart with unabsorbed colors suggestive of a Rayleigh-Jeans spectrum but whose flux is in disagreement with most existing models for the X-ray emission (Hulleman et al. 2000). In particular, the unabsorbed $f_{V}$ exceeds that calculated from our models for the thermal surface emission $\left(\log B=14, \log T_{\text {eff }}=6.5\right)$ by $\sim 5$ orders of magnitude. We can therefore exclude thermal surface emission as a significant contribution to the optical flux.

The recent report of optical pulsations at the level of $30 \%$, in phase with and having the same dual-peak structure as the X-ray pulsations (Kern \& Martin 2002), poses additional difficulties for unified models of the AXP spectra, suggesting that the optical and X-ray pulsations originate from a common source. It is unlikely that the other AXPs can be observed at optical frequencies in the foreseeable future because of their greater distances and heavily absorbed lines of sight.

\section{REFERENCES}

Arnaud, K. A. 1996, in ASP Conf. Ser. 101, Astronomical Data Analysis Software and Systems V, ed. G. H. Jacoby \& J. Barnes (San Francisco: ASP), 17

Bignami, G. F., Caraveo, P. A., \& Paul, J. A. 1988, A\&A, 202, L1

Brown, E. F., Bildsten, L., \& Rutledge, R. E. 1998, ApJ, 504, L95

Chatterjee, P., Hernquist, L., \& Narayan, R. 2000, ApJ, 534, 373

Chiu, H.-Y., \& Salpeter, E. E. 1964, Phys. Rev. Lett., 12, 413

Duncan, R. C., \& Thompson, C. 1992, ApJ, 392, L9

Golden, A., \& Shearer, A. 1999, A\&A, 342, L5

Gotthelf, E. V., Petre, R., \& Vasisht, G. 1999, ApJ, 514, L107

Heyl, J. S. \& Hernquist, L. 1998a, MNRAS, 300, 599 1998b, MNRAS, 298, L17

Heyl, J. S., \& Kulkarni, S. R. 1998, ApJ, 506, L61

Hulleman, F., van Kerkwijk, M. H., \& Kulkarni, S. R. 2000, Nature, 408, 689

Israel, G. L., et al. 1999, A\&A, 346, 929

Jacchia, A., de Luca, F., Lazzaro, E., Caraveo, P. A., Mignani, R. P., \& Bignami, G. F. 1999, A\&A, 347, 494

Juett, A. M., Marshall, H. L., Chakrabarty, D., \& Schulz, N. S. 2002, ApJ, 568, L31

Kern, B., \& Martin, C. 2002, Nature, 417, 527

Lai, D., \& Ho, W. C. G. 2002, ApJ, 566, 373

Lloyd, D. A. 2003, MNRAS, submitted (astro-ph/0303561)

Louden, R. 1959, Am. J. Phys., 27, 649

Martin, C., Halpern, J. P., \& Schiminovich, D. 1998, ApJ, 494, L211

Menou, K., Perna, R., \& Hernquist, L. 2001, ApJ, 559, 1032

Mereghetti, S., Mignani, R. P., Covino, S., Chaty, S., Israel, G. L., Neuhäuser, R., Plana, H., \& Stella, L. 2001, MNRAS, 321, 143

Mereghetti, S., \& Stella, L. 1995, ApJ, 442, L17

Mészáros, P. 1992, High-Energy Radiation from Magnetized Neutron Stars (Chicago: Univ. Chicago Press)

Mignani, R., Caraveo, P. A., \& Bignami, G. F. 1997, ApJ, 474, L51

Mihalas, D. 1978, Stellar Atmospheres (San Francisco: W. H. Freeman \& Co.)

Ögelman, H. B. 1995, in The Lives of the Neutron Stars, ed. M. A. Alpar, Ü. Kiziloğlu, \& J. van Paradijs (Dordrecht: Kluwer), 101

Ögelman, H., \& Finley, J. P. 1993, ApJ, 413, L31

Page, D. 1995, ApJ, 442, 273

Pavlov, G. G., Shibanov, Y. A., Zavlin, V. E., \& Meyer, R. D. 1995, in The Lives of the Neutron Stars, ed. M. A. Alpar, Ü. Kiziloğlu, \& J. van Paradijs (Dordrecht: Kluwer), 71

Pavlov, G. G., Stringfellow, G. S., \& Córdova, F. A. 1996, ApJ, 467, 370

Rajagopal, M., \& Romani, R. W. 1996, ApJ, 461, 327

Rajagopal, M., Romani, R. W., \& Miller, M. C. 1997, ApJ, 479, 347

Stella, L., Israel, G. L., \& Mereghetti, S. 1998, Adv. Space Res., 22, 1025

Thompson, C., \& Duncan, R. C. 1996, ApJ, 473, 322

Tsuruta, S. 1964, Ph.D. thesis, Columbia Univ.

van Paradijs, J., Taam, R. E., \& van den Heuvel, E. P. J. 1995, A\&A, 299, L41

Ventura, J. 1979, Phys. Rev. D, 19, 1684 